\title{
Arriving at the Right Diagnosis in an Era of Precision Medicine
}

\author{
Vina Pulido $^{a}$ Shin Yin Lee ${ }^{b} \quad$ Naomi Yu Ko ${ }^{b}$ \\ a Department of Medicine, Boston Medical Center, Boston, Mass., USA; \\ ${ }^{b}$ Section of Hematology/Oncology, Department of Medicine, Boston Medical Center, \\ Boston, Mass., USA
}

\section{Keywords}

Thyroid carcinoma $\cdot$ Targeted therapy

\section{Abstract}

In the era of precision medicine and targeted therapy, diagnostic inaccuracy can have tremendous ramifications. We present the case of a 61-year-old man initially diagnosed with small cell lung cancer by pathology. Prior to initiating chemotherapy, multidisciplinary discussions led to an amendment of the diagnosis to medullary thyroid cancer.

(C) 2016 The Author(s)

Published by S. Karger AG, Basel

\section{Introduction}

In the era of precision medicine and targeted therapy, diagnostic inaccuracy can have tremendous ramifications. When molecularly targeted agents for specific, and often rare, tumor types are available, obtaining the correct diagnosis is critical. Here, we demonstrate a case report of an individual with a challenging diagnosis of a rare tumor type. 


\section{Case Presentation}

A 61-year-old Polish man presented to the emergency department with gradually worsening shortness of breath and unintentional 20-pound weight loss. He had been in his usual state of health until 1 year prior to presentation, when he started to experience progressive exercise intolerance. He was prompted to seek medical attention when he started waking up at night with shortness of breath. He denied any associated edema, fever, chills, or night sweats. There was no other significant past medical history except remote treatment for latent tuberculosis. He was on no medications and had no known drug allergies. He was originally from Poland, was working as a cook, had smoked half a pack a day of cigarettes for 15 years (quit 30 years ago), and denied any illicit drug or alcohol use. The family history was significant for an older brother in Poland, who died in his 40s from a type of cancer unknown to him.

On admission, the patient was afebrile, his heart rate was in the $80 \mathrm{~s}$, his blood pressure ranged from 120 to 140 and 60 to $70 \mathrm{~mm} \mathrm{Hg}$, and oxygen saturation was $89 \%$ on room air and $93 \%$ on 2 liters of oxygen by nasal cannula. His respiratory rate was 16 breaths $/ \mathrm{min}$. A physical examination was notable for stridor and a diffuse, large, palpable neck mass greater on the right than on the left. His initial laboratory examinations, consisting of a complete blood count and basic metabolic panel, were remarkable only for mildly low hemoglobin $(12.5 \mathrm{~g} / \mathrm{dl})$. Chest X-ray showed a large mediastinal mass with bilateral hilar lymphadenopathy and partial collapse of the bilateral upper lobes (fig. 1). Computed tomography of the thorax with contrast revealed a partially necrotic, lobulated mass measuring $17.6 \times 9.5 \mathrm{~cm}$ in the anterior mediastinum with involvement of the thyroid gland and bilateral hila, encasing the superior vena cava. Numerous pulmonary nodules as well as mediastinal and supraclavicular lymphadenopathy were noted. There was also moderate narrowing of the trachea at the level of the thoracic inlet. A focal lytic lesion in the right posterolateral 6th rib was concerning for metastatic disease. Staging scans obtained shortly thereafter ruled out metastatic disease in the brain and abdomen/pelvis. Thyroid function tests were within normal limits.

By the end of hospital day 1, the patient's breathing had improved with supplemental oxygen and incentive spirometry. His dyspnea was felt to be secondary to the upper lung collapse from the mass. On hospital day 2 , two core biopsies of the mass were taken from a superficial right neck approach. Hematoxylin and eosin staining showed malignant small, round, blue cells with nuclear pleomorphism and fine nuclear chromatin as well as some spindle cells (fig. 2). Immunohistochemistry stains were positive for synaptophysin, cytokeratins AE1/AE3, thyroid transcription factor-1 (TTF-1), and chromogranin A. The fibrous tissue surrounding the tumor cells stained positive with Congo red stain. No necrotic debris was seen. The final pathology impression was small cell lung carcinoma (SCLC). The patient was transferred to the hematology/oncology floor on hospital day 4 to start chemotherapy for presumed extensive-stage SCLC.

Before chemotherapy was initiated, a closer examination of the patient's history, presentation, and clinical data raised doubt on the diagnosis of SCLC. First, the patient had only a remote 8-pack-year smoking history. Second, upon detailed review of the patient's radiographic images, the large mediastinal mass appeared to be directly invading the thyroid. Third, the slow onset of the patient's symptoms and evidence of extensive collateral vessels was indicative of an insidious disease onset, which is atypical of SCLC. Fourth, the patient's brother died at a young age from a tumor in the thorax that was never properly diagnosed. This suggested a possible familial cancer syndrome. Fifth, despite the large size of the mass, there was no evidence of metastatic disease outside of the thorax, which was again 
unusual for SCLC. Sixth, amyloid infiltration is not usually seen with SCLC, but is seen in medullary thyroid carcinoma (MTC). Lastly, MTC, while much rarer, could have a similar histologic appearance. These pieces of history, radiologic findings, and pathologic information raised the suspicion that he may in fact have a rare thyroid malignancy.

Serum carcinoembryonic antigen (CEA) and calcitonin levels were obtained, and they were markedly elevated at $>15,000 \mathrm{ng} / \mathrm{ml}$ and $12,135 \mathrm{pg} / \mathrm{ml}$, respectively. Additional immunohistochemical (IHC) staining returned positive for calcitonin and CEA, consistent with MTC (fig. 3). The patient's diagnosis was amended to MTC on hospital day 6. He also later tested negative for the germline RET proto-oncogene mutation.

\section{Discussion}

This case highlights the importance of correlating a patient's history, radiographic images, and biopsy results in order to arrive at an accurate oncologic diagnosis. Proper diagnosis has dramatic consequences in this era of personalized medicine with greater opportunities for targeted therapy. In this case, although the imaging and core biopsies in our patient were initially consistent with SCLC, his clinical presentation was atypical for this diagnosis. Recognizing this inconsistency led to further discussion with other providers (radiology and pathology) and testing for thyroid-specific IHC staining and tumor markers (CEA and calcitonin), which confirmed the diagnosis of MTC. This case highlights that while pathologic findings often dictate cancer diagnoses, ensuring that the clinical and radiographic findings are consistent with the tissue diagnosis is essential to accurate diagnosis and management.

MTC is a rare neuroendocrine malignancy of the parafollicular or C cells of the thyroid gland [1]. It accounts for 3-4\% of thyroid cancers in the USA [2]. Most cases are sporadic, but about $25 \%$ are hereditary, as part of the multiple endocrine neoplasia type 2 syndrome [3]. Although rare, MTC causes $15 \%$ of thyroid-related cancer deaths [4]. Patients with localized disease have a 10 -year survival rate of $95 \%$, compared to $75.5 \%$ for those with regional disease and $40 \%$ for those with distant metastases [5].

MTC has represented a diagnostic challenge in clinical practice, due in part to its rarity [6]. Fine needle aspiration of thyroid nodules is the gold standard for the diagnosis of MTC; yet its sensitivity was only $63 \%$ in one case series of 67 patients and $56.4 \%$ in a metaanalysis of 641 MTC lesions [6, 7]. False negatives were usually due to misinterpretation of MTC as follicular thyroid cancer or Hürthle cell cancer, but rarely as small cell cancer [7].

The pathologic diagnosis of MTC can be difficult, due to the variability in clinical presentation, size, and morphology. Our patient had adequate samples obtained via core biopsy, and was found to have malignant cells with fine nuclear chromatin, inconspicuous nucleoli, and sparse cytoplasm. IHC staining revealed positive staining for cytokeratin AE1/3, chromogranin, synaptophysin, and TTF-1. The microscopic findings are similar to what is seen in SCLC, which typically has small cells with fine granular nuclear chromatin and faint nucleoli. Typical IHC features of SCLC also include positivity for AE1/3, TTF-1, chromogranin, and synaptophysin [8].

Aside from the history as well as physical and radiological findings, specific serum tumor markers and IHC staining helped to confirm the diagnosis in our patient. His serum calcitonin level was profoundly elevated at $12,135 \mathrm{pg} / \mathrm{ml}$. The tumor stained positive for calcitonin as well. Calcitonin is produced by the $\mathrm{C}$ cells of the thyroid, and there is evidence that measurement of levels in patients with thyroid nodules can lead to earlier diagnosis of MTC [9]. The serum calcitonin level is not a specific marker of MTC, but it has been found to be 
more sensitive than fine needle aspiration cytology in the preoperative diagnosis of this disease (98 vs. 63\%) [6]. There exists a small cell variant of MTC, which histologically and cytologically resembles SCLC or malignant lymphoma, and may not necessarily stain for calcitonin [10]. CEA is a biomarker of several cancers, but is typically overexpressed in MTC [11]. Serum CEA can be elevated in advanced stages of MTC, and may assist in the diagnostic workup. Our patient's tumor was immunochemically sensitive for CEA, and he also had an impressively high serum CEA level of $>15,000 \mathrm{ng} / \mathrm{ml}$.

In the current era of precision medicine, targeted therapy for specific tumor mutations can have dramatic results. In the past, treatment of MTC consisted of systemic chemotherapy, including 5-fluorouracil, dacarbazine, and anthracyclines, with poor response rates of about $10-20 \%[12,13]$. The discovery of tyrosine kinase activation has revolutionized treatment of MTC. In addition to the RET mutation, hepatocyte growth factor receptor and vascular endothelial growth factor receptor 2 signaling pathways have been identified to be upregulated in thyroid tumors. These pathways have become the target for small-molecule tyrosine kinase inhibitors such as vandetanib [14] and cabozantinib [15], demonstrating dramatic results.

In conclusion, we report a case of aggressive medullary thyroid cancer extending into the mediastinum whose initial pathology and diagnosis was SCLC. This case highlights that the cytologic features of SCLC and MTC can be similar, and misdiagnosis is possible. With careful analysis of our patient's presentation, history, examination, and imaging, the proper diagnosis of MTC was identified. Although a rare disease, simple additional testing of serum CEA and serum calcitonin, as well as IHC staining of the tumor for calcitonin and CEA, helped make the accurate diagnosis. Proper diagnosis of MTC has significant implications in the era of targeted therapy, and establishing an accurate diagnosis is vital to ensuring proper benefit from these new agents.

\section{Conclusion}

Proper diagnosis of rare tumors can be challenging, but it can yield unique opportunities for personalized treatment that has defined recent therapeutic discoveries in oncology.

\section{Statement of Ethics}

Consent to the publication of this manuscript was obtained.

\section{Disclosure Statement}

The authors declare no conflicts of interest.

\section{References}

1 Ferreira CV, Siqueira DR, Ceolin L, Maia AL: Advanced medullary thyroid cancer: pathophysiology and management. Cancer Manag Res 2013;5:57-66.

2 Davies L, Welch HG: Increasing incidence of thyroid cancer in the United States, 1973-2002. JAMA 2006;295:2164-2167. 
-3 Wells SA Jr, Asa SL, Dralle H, Elisei R, Evans DB, Gagel RF, et al: Revised American Thyroid Association guidelines for the management of medullary thyroid carcinoma. Thyroid 2015;25:567-610.

-4 Ahmed SR, Ball DW: Clinical review: incidentally discovered medullary thyroid cancer: diagnostic strategies and treatment. J Clin Endocrinol Metab 2011;96:1237-1245.

5 Roman S, Lin R, Sosa JA: Prognosis of medullary thyroid carcinoma: demographic, clinical, and pathologic predictors of survival in 1,252 cases. Cancer 2006;107:2134-2142.

-6 Bugalho MJ, Santos JR, Sobrinho L: Preoperative diagnosis of medullary thyroid carcinoma: fine needle aspiration cytology as compared with serum calcitonin measurement. J Surg Oncol 2005;91:56-60.

7 Trimboli P, Treglia G, Guidobaldi L, Romanelli F, Nigri G, Valabrega S, et al: Detection rate of FNA cytology in medullary thyroid carcinoma: a meta-analysis. Clin Endocrinol (Oxf) 2015;82:280-285.

-8 Travis WD: Update on small cell carcinoma and its differentiation from squamous cell carcinoma and other non-small cell carcinomas. Mod Pathol 2012;25(suppl 1):S18-S30.

-9 Costante G, Meringolo D, Durante C, Bianchi D, Nocera M, Tumino S, et al: Predictive value of serum calcitonin levels for preoperative diagnosis of medullary thyroid carcinoma in a cohort of 5,817 consecutive patients with thyroid nodules. J Clin Endocrinol Metab 2007;92:450-455.

10 Yerly S, Triponez F, Meyer P, Kumar N, Bongiovanni M: Medullary thyroid carcinoma, small cell variant, as a diagnostic challenge on fine needle aspiration: a case report. Acta Cytol 2010;54(suppl):911-917.

11 Behr TM, Gratz S, Markus PM, Dunn RM, Hufner M, Schauer A, et al: Anti-carcinoembryonic antigen antibodies versus somatostatin analogs in the detection of metastatic medullary thyroid carcinoma: are carcinoembryonic antigen and somatostatin receptor expression prognostic factors? Cancer 1997;80(suppl):2436-2457.

$\$ 12$ Orlandi F, Caraci P, Berruti A, Puligheddu B, Pivano G, Dogliotti L, et al: Chemotherapy with dacarbazine and 5-fluorouracil in advanced medullary thyroid cancer. Ann Oncol 1994;5:763-765.

-13 Wu LT, Averbuch SD, Ball DW, de Bustros A, Baylin SB, McGuire WP 3rd: Treatment of advanced medullary thyroid carcinoma with a combination of cyclophosphamide, vincristine, and dacarbazine. Cancer 1994;73:432-436.

-14 Wells SA Jr, Robinson BG, Gagel RF, Dralle H, Fagin JA, Santoro M, et al: Vandetanib in patients with locally advanced or metastatic medullary thyroid cancer: a randomized, double-blind phase III trial. J Clin Oncol 2012;30:134-141.

15 Elisei R, Schlumberger MJ, Müller SP, Schöffski P, Brose MS, Shah MH, et al: Cabozantinib in progressive medullary thyroid cancer. J Clin Oncol 2013;31:3639-3646. 


\section{Case Reports in Oncology}

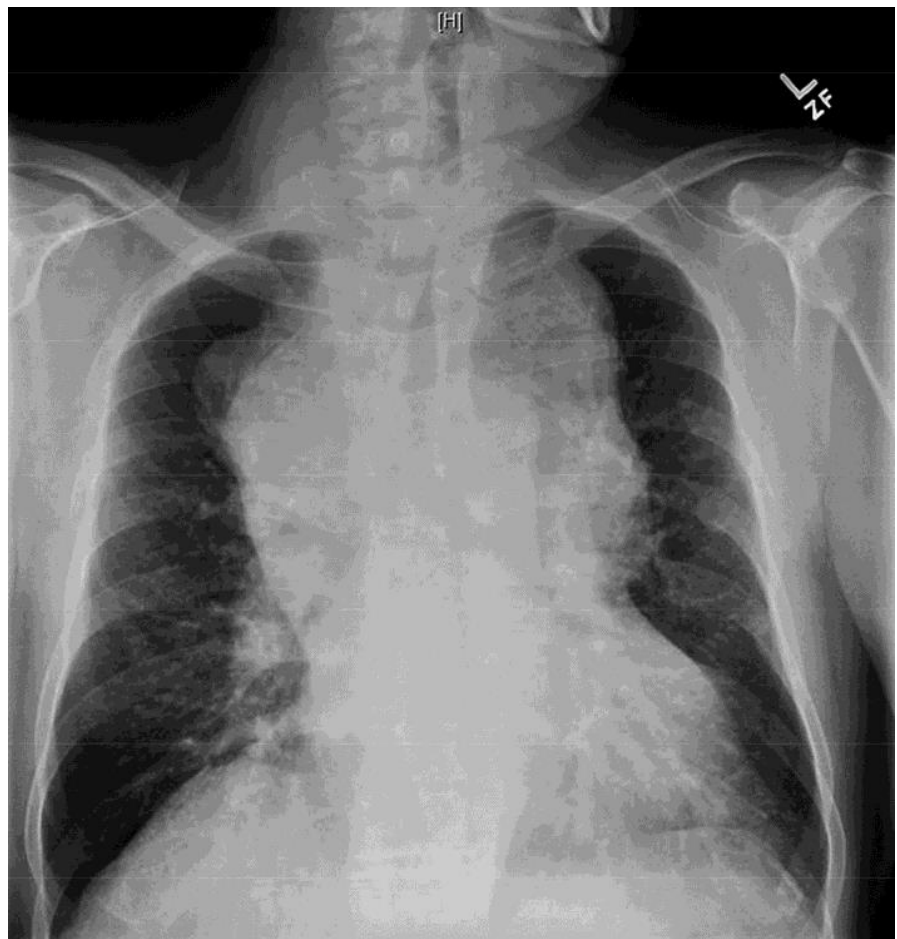

Fig. 1. Chest X-ray on presentation.

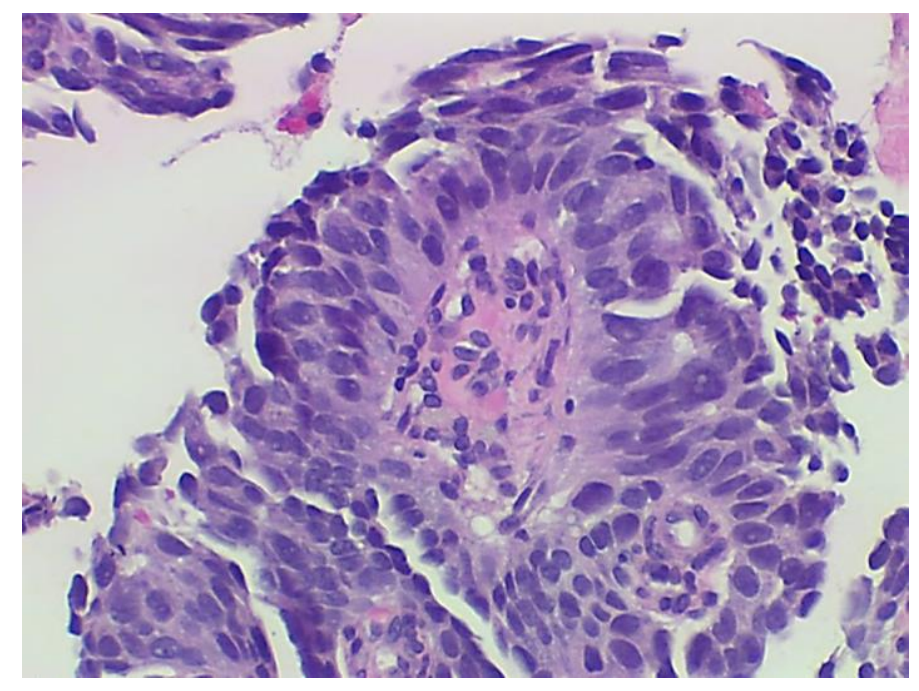

Fig. 2. Initial hematoxylin and eosin IHC stain. 


\section{Case Reports in \begin{tabular}{l|l}
\multicolumn{3}{c}{ Case Rep Oncol 2016;9:351-357 } \\
\hline $10.1159 / 000447351$ & (c) 2016 The Author(s). Published by S. Karger AG, Basel
\end{tabular} Oncology www.karger.com/cro \\ Pulido et al.: Arriving at the Right Diagnosis in an Era of Precision Medicine}

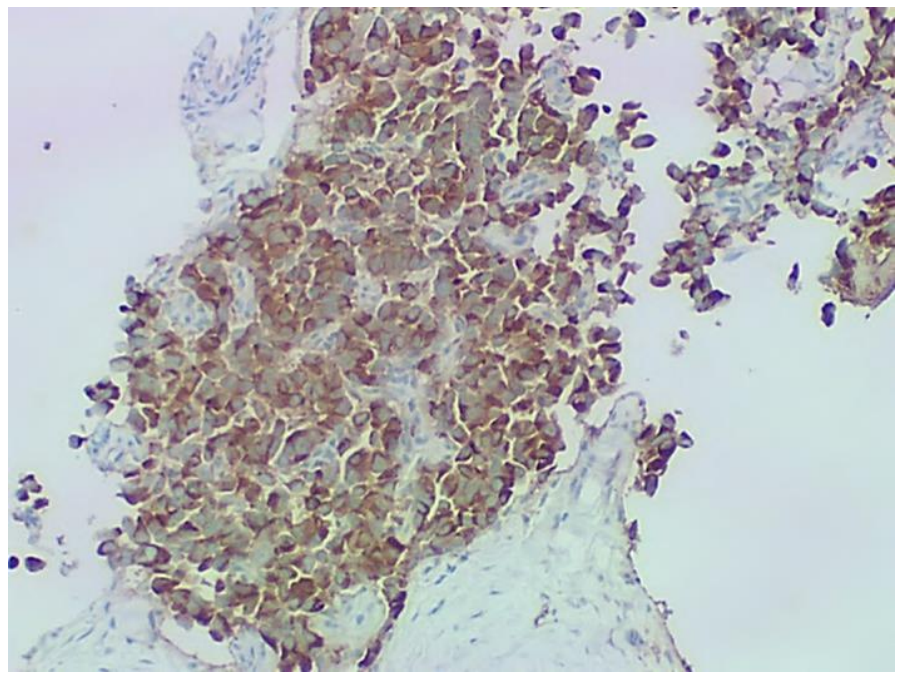

Fig. 3. Calcitonin and CEA IHC stain. 